\title{
Social misdirection fails to enhance a magic illusion
}

\author{
Jie Cui ${ }^{1}$, Jorge Otero-Millan ${ }^{1,2}$, Stephen L. Macknik ${ }^{1,3}$, Mac King $^{1}$ and Susana Martinez-Conde ${ }^{1}$ * \\ ' Division of Neurobiology, Barrow Neurological Institute, Phoenix, AZ, USA \\ 2 Signal Theory and Communications, University of Vigo, Vigo, Spain \\ ${ }^{3}$ Division of Neurosurgery, Barrow Neurological Institute, Phoenix, AZ, USA
}

Edited by:

Luis M. Martinez, CSIC - Universidad

Miguel Hernandez, Spain

Reviewed by:

Anthony S. Barnhart, Arizona State

University, USA

Daniel J. Simons, University of

Illinois, USA

\section{*Correspondence.}

Susana Martinez-Conde, Laboratory of Visual Neuroscience, Division of Neurobiology, Barrow Neurological Institute, 350 West Thomas Road, Phoenix, AZ 85013, USA.

e-mail: smart@neuralcorrelate.com
Visual, multisensory and cognitive illusions in magic performances provide new windows into the psychological and neural principles of perception, attention, and cognition. We investigated a magic effect consisting of a coin "vanish" (i.e., the perceptual disappearance of a coin after a simulated toss from hand to hand). Previous research has shown that magicians can use joint attention cues such as their own gaze direction to strengthen the observers' perception of magic. Here we presented naïve observers with videos including real and simulated coin tosses to determine if joint attention might enhance the illusory perception of simulated coin tosses. The observers' eye positions were measured, and their perceptual responses simultaneously recorded via button press. To control for the magician's use of joint attention cues, we occluded his head in half of the trials. We found that subjects did not direct their gaze at the magician's face at the time of the coin toss, whether the face was visible or occluded, and that the presence of the magician's face did not enhance the illusion. Thus, our results show that joint attention is not necessary for the perception of this effect. We conclude that social misdirection is redundant and possibly detracting to this very robust sleight-of-hand illusion. We further determined that subjects required multiple trials to effectively distinguish real from simulated tosses; thus the illusion was resilient to repeated viewing.

Keywords: join attention, social misdirection, fixation, free-viewing, eye movements, sleight-of-hand, prestidigitation, motion perception

\section{INTRODUCTION}

Visual, multisensory, and cognitive illusions in magic performances provide new windows into the psychological and neural principles of perception, attention, and cognition (Macknik et al., 2008, 2010; Martinez-Conde and Macknik, 2008). Here we investigated a magic effect consisting of a coin "vanish" (i.e., the perceptual disappearance of a coin after a simulated toss from hand to hand).

A professional magician (Mac King, headliner, Harrah's Las Vegas) performed the coin vanish, as follows: (a) The magician tosses the coin vertically in his right hand; (b) The magician pretends to toss the coin from right to left hand, but surreptitiously holds the coin in his right hand, stopping it from flying; (c) The magician's left hand closes as if "catching" the supposedly flying coin; (d) The magician opens his left hand to show that the coin has disappeared (Figure 2A; Video S1 in Supplementary Material). Naïve observers typically perceive the coin flying from right to left hand, and are surprised to find the coin "magically" gone when the magician opens his left hand.

Magicians often perform this particular coin vanish while directing their own gaze to the presumed position of the coin at any given time, never looking at the spectators directly. Previous research has shown that magicians can use joint attention cues such as their own gaze direction to strengthen the observers' perception of magic, however (i.e., in the Vanishing Ball illusion; Kuhn and Land, 2006).

We wondered if Mac King might similarly enhance the present illusion by raising his eyes to face the viewer at the time of the simulated coin toss. If observers responded to Mac King's social misdirection by returning his gaze at the time of the toss, they would necessarily view the toss with their peripheral vision. If so, their perception of the toss might be enhanced (i.e., they would see the simulated toss with lower spatial resolution, in a part of the visual field where neurons are especially sensitive to motion cues; Hubel, 1988).

We presented naïve observers with videos of Mac King performing real and simulated coin tosses, with the magician's head occluded in half of the trials to control for the magician's use of his own gaze as an element of misdirection. We further included fixation trials (in which subjects were forced to look at the magician's face) and free-viewing trials (in which subjects were allowed to explore the scene freely, as they would during a magic show) to study the effect of peripheral versus central viewing on the perception of the illusion. Finally, we presented the subjects with both real and simulated tosses multiple times, to determine the effect of repeated viewing on the perception of this illusion. The subjects' eye positions were simultaneously measured, and their perceptual responses recorded via button press (see Materials and Methods: General for details).

\section{MATERIALS AND METHODS: GENERAL SUBJECTS}

All subjects were naïve, had normal or corrected-to-normal vision, and were paid $\$ 15$ for a single experimental session ( $\sim 60 \mathrm{~min}$ ). Experiments were carried out under the guidelines of the Barrow Neurological Institute's Institutional Review Board (protocol 


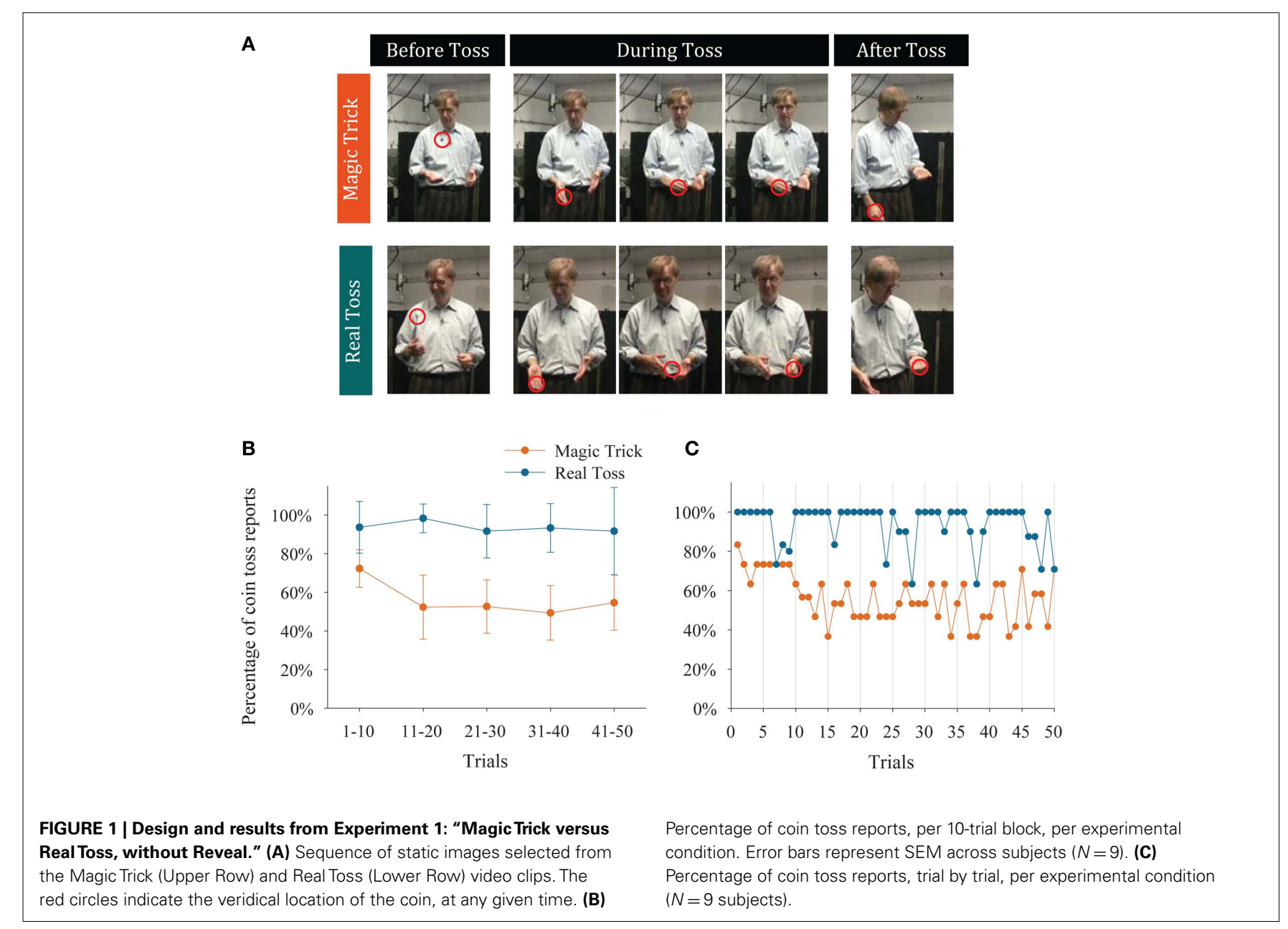

04BN039) and written informed consent was obtained from each participant. Nine subjects ( 3 females, 6 males) participated in Experiment 1. Six new subjects ( 2 females, 4 males) participated in Experiment 2. Eight new subjects ( 1 female, 7 males) participated in Experiment 3.

\section{EYE MOVEMENTS RECORDINGS}

Subjects rested their head on a chin/forehead-rest $57 \mathrm{~cm}$ from a video monitor (Barco Reference Calibrator V, 60-Hz refresh rate).

Eye position was acquired non-invasively with a fast videobased eye movement monitor (EyeLink 1000, SR Research) at 500 samples per second (instrument noise $0.01^{\circ} \mathrm{rms}$ ). We identified and removed blink periods as the portions of the EyeLink 1000 recorded data where the pupil information was missing. We added $200 \mathrm{~ms}$ before and after each EyeLink 1000 identified blink period to further eliminate the initial and final parts of the blink, where the pupil is only partially occluded. Eye positions during the blinks were calculated by linear interpolation from the eye positions at the beginning of the blink to the end of the blink (Bour et al., 2000).

\section{VISUAL STIMULI}

All videos had the same frame size $\left[26.7^{\circ}(\mathrm{w}) \times 14.1^{\circ}(\mathrm{h})\right]$ and were displayed centrally on the monitor screen $\left[40^{\circ}(\mathrm{w}) \times 30^{\circ}(\mathrm{h})\right]$.
Video clips S1-S5 in Supplementary Material may be divided into four segments: (1) "Before Toss" refers to the performance from the start of the video to the initiation of "Toss." During this period, the magician either throws a coin up in the air, using his right hand (as in Videos S1-S3, S6, and S7 in Supplementary Material) or pretends to do so (Videos S4 and S5 in Supplementary Material); (2) "During Toss" is the period from toss initiation to the end of the toss (i.e. beginning of "After Toss"). The "Toss" can be either a "Real Toss," i.e., the magician throws a coin from right to left hand (Videos S2 and S7 in Supplementary Material) or a "Fake Toss," i.e., the magician either pretends to throw the coin from right to left hand, but surreptitiously retains it in his right hand (Videos S1, S3, and S6 in Supplementary Material), or simply performs a tossing gesture (i.e., without a coin) from right to left hand (Videos S4 and S5 in Supplementary Material); (3) "After Toss" extends from the end of toss to the opening of the magician's left hand; and (4) "Reveal" from the opening of the left hand to the end of the video. That is, the "Reveal" stage starts at the time the magician first opens his left hand, and ends with the last frame of the performance.

Videos S6 and S7 in Supplementary Material are identical to Videos S1 and S2 in Supplementary Material, respectively, except that the "Reveal" segments are omitted. 


\section{DATA ANALYSES}

\section{Perceptual reports analysis}

In Experiment 1 and Experiment 2, we calculated the percentage of coin toss reports (i.e., the percentage of times the subject saw the coin flying from right to left hand) in each block of 10 consecutive trials of the same condition (Figures $\mathbf{1 B}$ and $\mathbf{2 B}$ ), and on a trial-by-trial basis (Figures 1C and 2C), for each subject. We then calculated the average and the SD across the subjects.

\section{Gaze dynamics analysis}

In Experiment 3, we considered that subjects looked at the face of the magician if their gaze entered a circular area with a $3^{\circ}$ diameter, centered on the midpoint between the magician's eyes, for at least one data sample.

To obtain the colormaps in Figure 8, we added the amount of time that subjects allocated their gaze to every pixel of the screen from the beginning to the end of the toss. Colormaps were smoothed using a $40 \times 40$ pixel $\left(\sim 1.2^{\circ} \times 1.2^{\circ}\right)$ Gaussian filter with a SD of 10 pixels $\left(\sim 0.3^{\circ}\right)$.

\section{Signal detection analyses}

We carried out analyses based on signal detection theory (Macmillan and Creelman, 2005) to investigate detection sensitivity and response bias in the three experiments conducted. All three experiments may be identified as single-interval classification experiments (Macmillan and Creelman, 2005). In Experiments 1 and 2 subjects used two responses (pressing or not pressing the button) to sort two stimulus conditions (Magic Trick and Real Toss) into categories. In Experiment 3, the subjects classified five stimulus conditions (Real Toss, Magic Trick, No Coin Fake Toss, Final Coin Fake Toss and Two Coins Fake Toss) into two categories by pressing or not pressing the button. Throughout the experiments, only one stimulus condition was presented in each trial.

In order to estimate the parameters from the signal detection model in a single-interval paradigm, we fitted the model for each subject with RscorePlus (Harvery, 2011). In the model, the output of sensory process under each of the $m$ stimulus conditions has a normal density function with mean $\mu_{j}$ and $\operatorname{SD} \sigma_{j}$, where $j=0, \ldots, m-1, \mu_{0}=0$, and $\sigma_{0}=1$. The model also assumes that subjects hold $n-1$ decision criteria $X_{c}$ to classify the output of the sensory process into $n$ response categories. The detection sensitivity for discriminating between two stimulus conditions is the absolute difference between the means of the two distributions, $d^{\prime}=\left|\mu_{j_{1}}-\mu_{j_{2}}\right|$, where $j_{1}, j_{2}=0,1, \ldots, m-1$ and $j_{1} \neq j_{2}$. RscorePlus employs singular value decomposition (Press, 2002), combined with a variation of the Marquardt method for non-linear
A
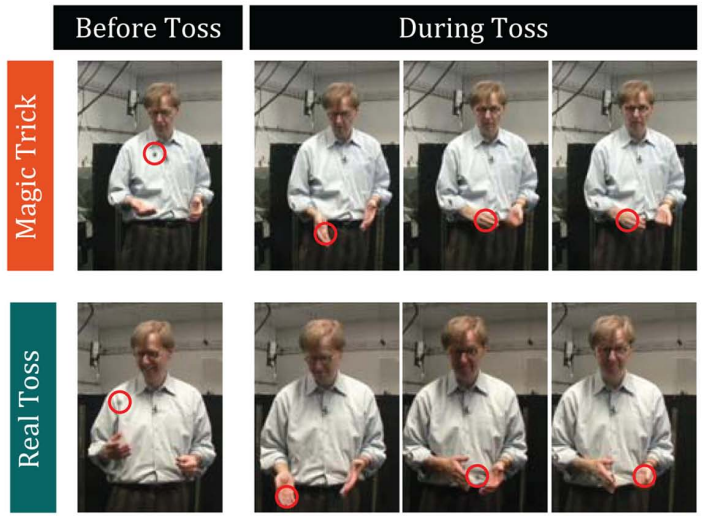

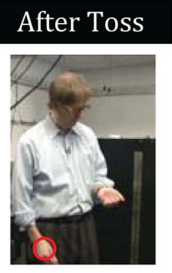

Reveal
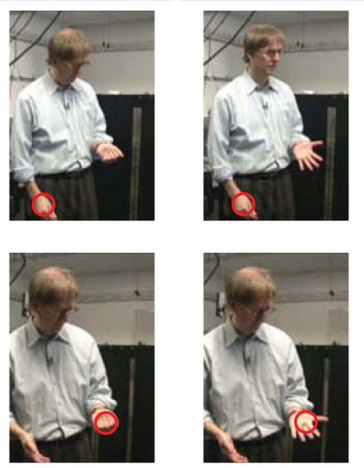

C
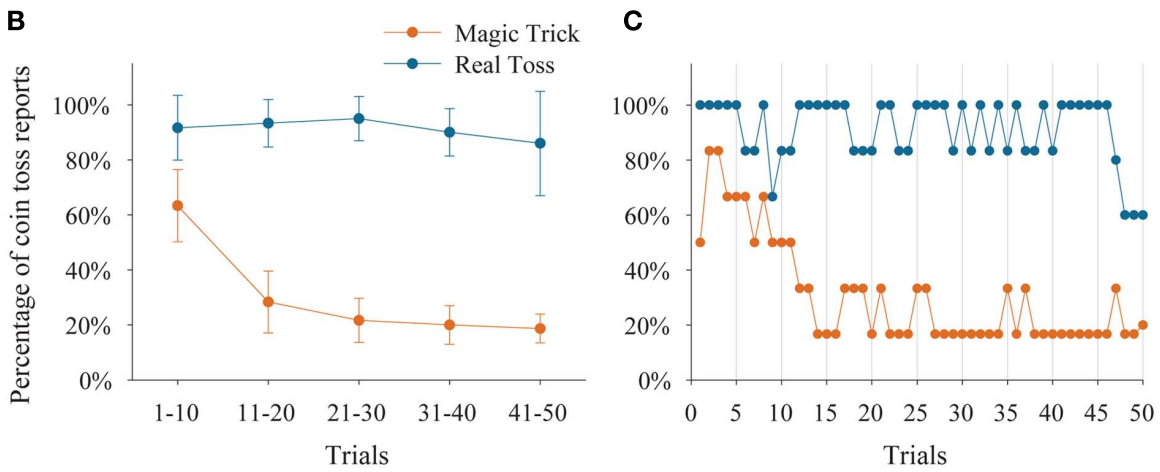

red circles indicate the veridical location of the coin, at any given time. (B) Percentage of coin toss reports, per trial block, per experimental condition. Error bars represent SEM across subjects $(N=8)$. (C) Percentage of coin toss reports, trial by trial, per experimental condition $(N=8$ subjects) 
least-squares regression (Marquardt, 1963; Press, 2002), to find the maximum-likelihood fit of the multiple-distribution, variablecriterion signal detection model. (Specifically, it finds the means $\mu_{j}$ and $\mathrm{SD} \sigma_{j}$ of the remaining signal distributions, $j=1, \ldots, m-1$, and then the decision criteria $X_{c}$ relative to the first signal distribution). In our case, the input data for RscorePlus was the percentage of trials with or without coin toss reports for each stimulus condition. The detection sensitivity determined if subjects were better at classifying in some stimulus conditions than in others. The decision criteria $X_{c}$ provided the response bias that indicated the subjects' tilt toward one response or the other (Macmillan and Creelman, 2005). Subsequently, we calculated the average and standard error of mean (SEM) from the measures of all subjects. During model fitting, we arbitrarily set the mean of the distribution of Real Toss at zero and found the means of remaining stimulus conditions relative to this position.

\section{EXPERIMENT 1 (MAGIC TRICK VERSUS REAL TOSS, WITHOUT REVEAL) \\ EXPERIMENT DESIGN}

Subjects pressed a key to start each trial. A blank screen lasting for $2 \mathrm{~s}$ was followed by a short video clip of Mac King performing one of two maneuvers: Magic Trick or Real Toss. Both videos stopped before the magician revealed the inside of his left hand (Figure 1A; Videos S6 and S7 in Supplementary Material). Subjects were asked to press a button, as soon as possible, in the event that they saw a coin flying from the magician's right hand to his left hand.

Each condition (Magic Trick or Real Toss) was presented for 50 trials, amounting to 100 trials in a single session. Trials were pseudo-randomly interleaved.

\section{RESULTS}

When Mac King actually tossed the coin from right to left hand (Real Toss), subjects indicated that they saw the coin toss $93.5 \%$ of the time, throughout the 50 trials presented (Figure 1B). When Mac King only pretended to toss the coin (Magic Trick), subjects reported at first a coin toss $72.6 \%$ of the time (averaged across the first 10 trials). This initial percentage dropped to $52.4 \%$ for the rest of the trials, reflecting the effects of the subjects' learning to discriminate between real and illusory coin tosses (the subjects performance in the first 10 trials was significantly different from that in the remaining 40 trials; two-sample $t$-test, $p<0.01$ ). Thus, subjects were able to distinguish between the two experimental conditions with maximal sensitivity after about 10 trials of each condition (that is, 20 trials combined). Figure 1C illustrates on a trial-by-trial basis the percentage of times that a coin toss was reported. Despite significant variance in the subjects' responses across time, the percentage of coin toss reports decreased steadily for the first few trials, stabilizing at around trial number 10 .

\section{EXPERIMENT 2 (MAGIC TRICK VERSUS REAL TOSS, WITH REVEAL) \\ EXPERIMENT DESIGN}

Experiment 2 followed the design of Experiment 1, except that both video clips (Magic Trick and Real Toss) now included an additional "Reveal" stage, where the magician opened his left hand to reveal that it was empty (Figure 2A; Videos S1 and S2 in Supplementary Material).

\section{RESULTS}

When Mac King actually tossed the coin from right to left hand (Real Toss with Reveal), subjects indicated that they saw the coin toss as often as in the equivalent condition (Real Toss without Reveal) from Experiment 1 (91.3\% of the time, throughout the 50 trials presented; Figure 2B). When Mac King only pretended to toss the coin (Magic Trick with Reveal), subjects reported at first a coin toss around $62.9 \%$ of the time (averaged across the first 10 trials). This initial percentage was not significantly different from that obtained in the equivalent condition (Magic Trick without Reveal) from Experiment 1 (paired $t$-test, $p>0.05$ ). The next 40 trials represented a distinct departure from the results from Experiment 1, however, as the coin toss reports dropped to $21.9 \%$ (a significant difference from the matching data in Experiment 1 ; paired $t$-test, $p<0.001)$. Thus including a "Reveal" stage with each performance allowed the subjects to gain additional information after about 10 trials of each condition (that is, 20 trials combined), thereby improving their discrimination of a real coin toss versus a simulated maneuver. Figure $2 \mathrm{C}$ illustrates on a trialby-trial basis the percentage of times that a coin toss was reported in Experiment 2.

Detection sensitivity and response bias were comparable in Experiment 1 (No Reveal) and Experiment 2 (Reveal) when we considered all 50 trials together, suggesting no effect of the "Reveal" stage (Figure 3, Left Column; see Materials and Methods: General for details on signal detection analyses). However, when we separated the first 10 trials from the last 40 trials, we found that the presence of a "Reveal" stage led to a significant increase in detection sensitivity $\left(\mathrm{d}^{\prime}\right)$ in the last 40 trials (Figure 3, Right Column). This result suggests that the information obtained from the "Reveal" stage helped subjects to discriminate between real and simulated tosses, after a number of trials.

\section{EXPERIMENT 3 (MULTIPLE TOSS CONDITIONS, WITH REVEAL) EXPERIMENT DESIGN}

Experiment 3 followed the design of Experiment 2, except that we presented 5 different video clips: Magic Trick, Real Toss, Two Coins Fake Toss, Final Coin Fake Toss, and No Coin Fake Toss (Figure 4A; Videos S3-S5 in Supplementary Material). The Magic Trick and Real Toss video clips were identical to those in Experiment 2 . The three additional video clips portrayed the following maneuvers: In the Two Coins Fake Toss, Mac King pretended to toss a coin from right to left hand, but actually retained it in his right hand. Subsequently, he opened his left hand to reveal a second coin that had remained hidden (in the left hand) until the reveal. In the Final Coin Fake Toss, Mac King pretended to toss a nonexistent coin from right to left hand, and subsequently opened his left hand to reveal an actual coin (which had remained hidden in his left hand until that point). In the No Coin Fake Toss, Mac King pretended to toss a non-existent coin from right to left hand, and subsequently opened his left hand to reveal that it was empty.

Two main motivations of this experiment were to determine the potential effects of (a) the magician's use of social misdirection cues and (b) the observer's gaze position on the perception of this magic trick. Thus, each of the videos was presented with (a) the magician's face visible and occluded, and (b) two different viewing 


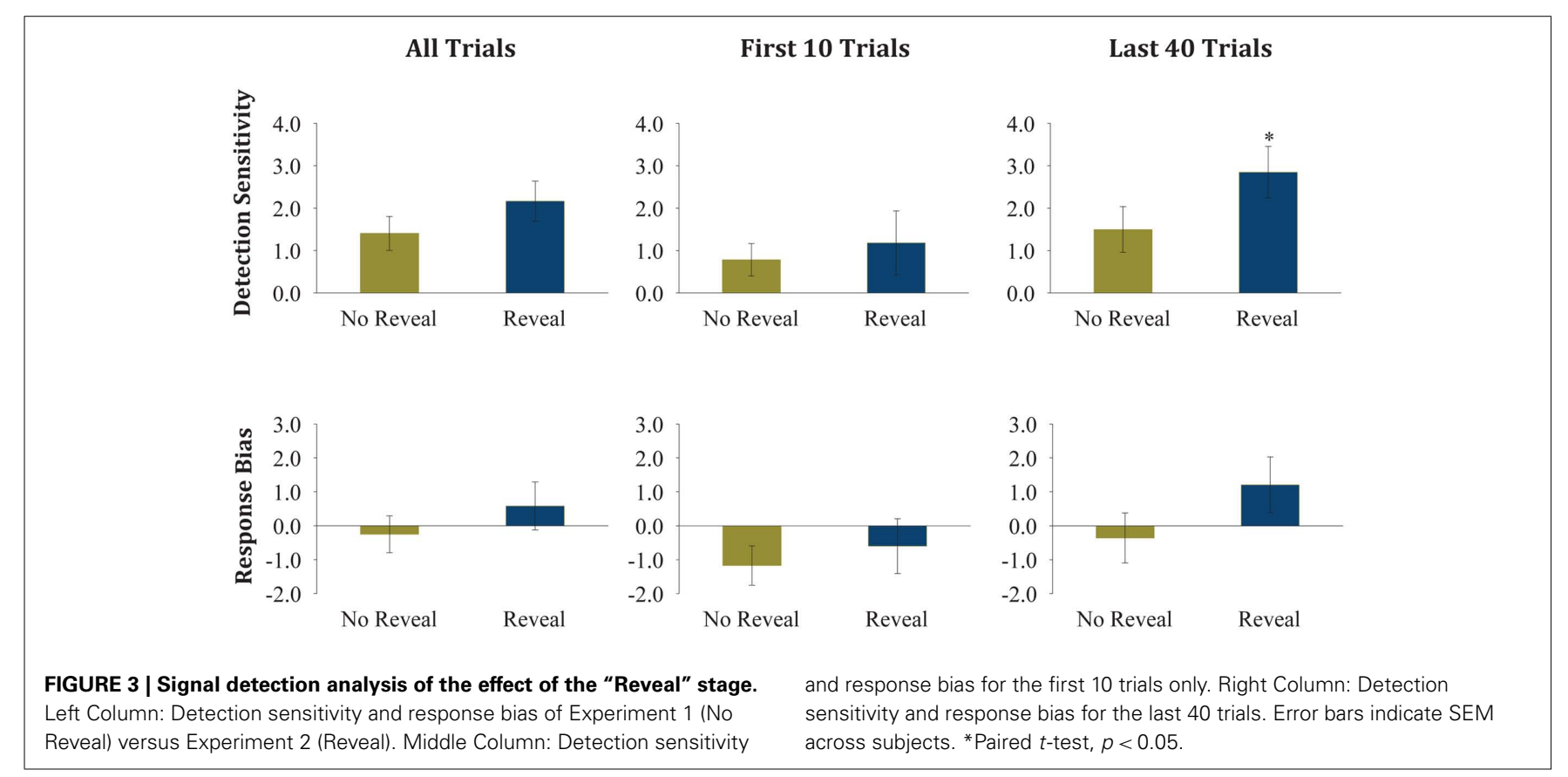

conditions: free-viewing and fixation (Figure 4B), for a total of 20 conditions ( 5 types of video clip $\times 2$ face conditions $\times 2$ viewing conditions). We presented 5 trials for each condition, amounting to 100 trials, pseudo-randomly ordered, in a single session.

In the Face Occluded condition, the face of the magician and surrounding area was blocked by an $11.9^{\circ}(\mathrm{w}) \times 5.6^{\circ}(\mathrm{h})$ black rectangle. In the Face Visible condition, no occlusion was used.

In the Fixation condition, subjects had to fixate a small red cross $\left(0.75^{\circ}\right.$ wide $)$ within a $2^{\circ} \times 2^{\circ}$ fixation window, placed in the midpoint between the magician's eyes (in the first frame the magician oriented his gaze to the viewer), or on the corresponding location in space when the magician's face was occluded. This fixation window was invisible to the subjects. A trial was discarded whenever the subject's gaze left the fixation window for more than $500 \mathrm{~ms}(<500 \mathrm{~ms}$ gaze excursions were permitted to allow for blinks). Then the discarded trial was inserted randomly into the subsequent queue of trials and presented to the subject again.

In the Free-viewing condition, no fixation cross was presented and subjects were free to explore the visual scene at will. Before each trial, we presented an instructions screen indicating whether fixation or free-viewing would be required.

\section{RESULTS}

The strength of the illusion was remarkable: subjects perceived illusory coin tosses a large fraction of the time, including during those trials in which the magician never showed a coin in the initial hand (Final Coin Fake Toss and No Coin Fake Toss conditions; Figure 5).

\section{Effect of social misdirection}

In each type of performance (Magic Trick, Real Toss, Two Coins Fake Toss, Final Coin Fake Toss, and No Coin Fake Toss), the magician raised his gaze to face the viewer at the time of the (real or simulated) coin toss. Our reasoning was that, if Mac King looked directly at the observers, he might engage them to reciprocate his gaze, thereby forcing them to view the coin toss peripherally, rather than foveally. If so, the subjects might perceive the illusion more strongly, especially as peripheral receptive fields are known to be more responsive to movement than foveal receptive fields (Hubel, 1988). Surprisingly, subjects reported higher percentages of coin tosses (Face visible $=72.4 \%$ versus Face Occluded $=88.4 \%$ ) when the magician's face was blocked (therefore nullifying the possibility of gaze misdirection) than when the magician's face was visible, across all types of performance (Figure 5).

The illusory effect was also stronger in absence of the magician's face when all five types of performance (Magic Trick, Real Toss, Two Coins Fake Toss, Final Coin Fake Toss, and No Coin Fake Toss) were grouped together, both under free-viewing and fixation conditions (Figure 6A).

We wondered if occluding the face of the magician could increase sensitivity or produce a criterion shift. We found a significant difference in response bias (but not in detection sensitivity) between the conditions of Face Visible and Face Occluded, indicating that subjects changed their criterion as a function of face visibility and were more likely to report a coin toss in the occluded face condition (Figure 7).

\section{Presence of an initial coin}

Experiments 1 and 2 showed that the illusion evoked by the simulated coin toss (Magic Trick condition) was very powerful and resilient to the effects of training (i.e., the subjects required about 10 observations of a simulated coin toss versus a real coin toss to differentiate one condition from the other optimally (Figures 1B and $2 \mathbf{B}$ ). We wondered whether the subjects might decide that a coin toss was real even before the (veridical or simulated) toss itself, based on the initial presence of a coin (as in the Magic Trick and Real Toss conditions, the coin toss is preceded by the magician throwing the coin up in the air and catching it in his right hand; 


\section{A}
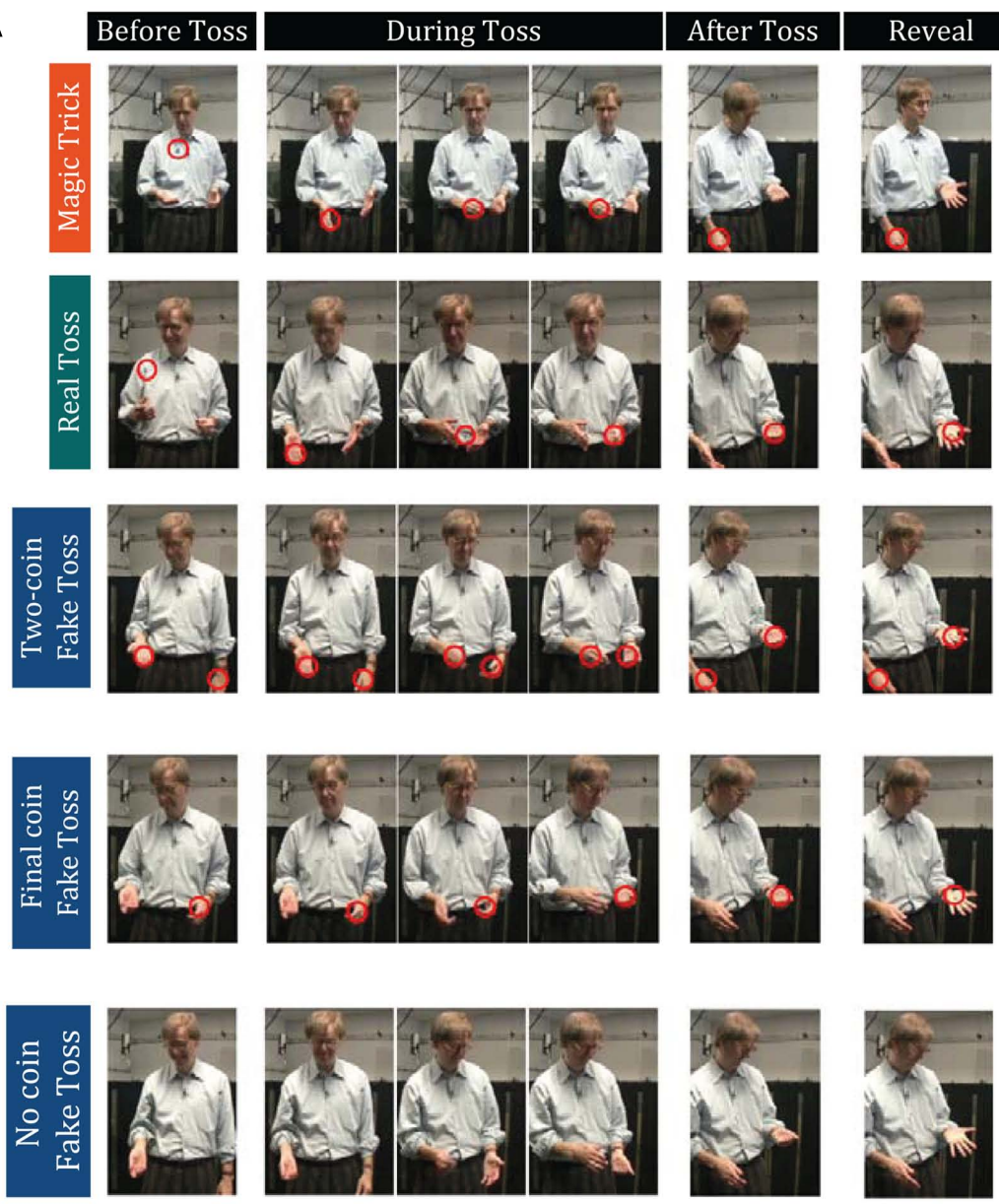

B

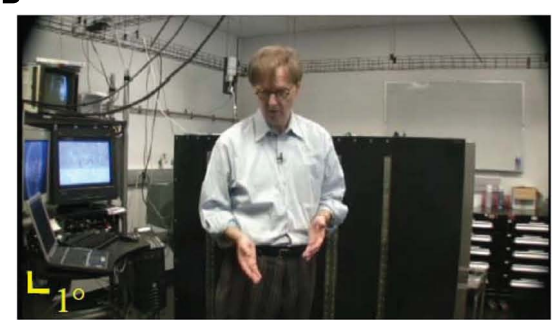

Free-viewing and Face Visible

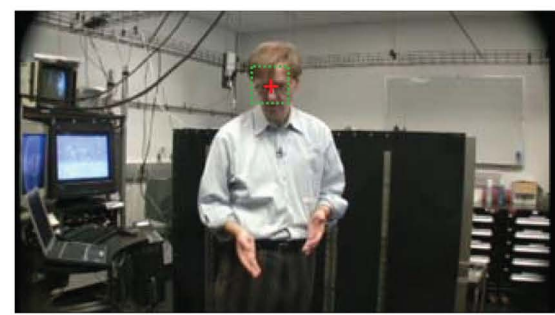

Fixation and Face Visible

FIGURE 4 | Design of Experiment 3: “Multiple Toss Conditions, with Reveal." (A) Sequence of static images selected from the various coin toss conditions presented: Magic Trick, Real Toss, Two-Coin Fake Toss, Final Coin Fake Toss, and No Coin Fake Toss. (B) Upper and Lower Rows:

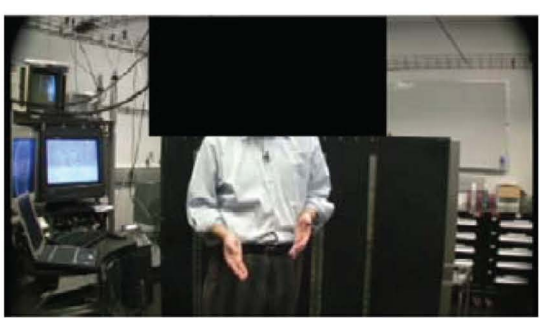

Free-viewing and Face Occluded

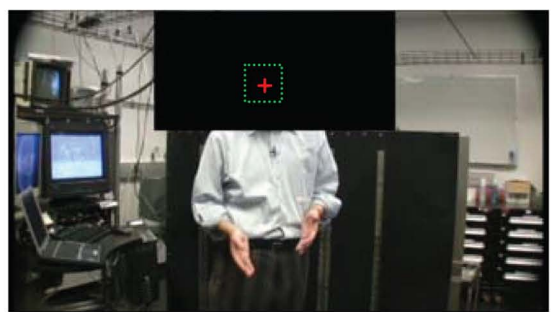

Fixation and Face Occluded

Free-exploration (Upper Row) and fixation (Lower Row) viewing conditions. Green dotted boxes (Lower Row) indicate the $2^{\circ} \times 2^{\circ}$ fixation window; fixation cross not to scale. Left and Right Columns: Magician's face visible (Left Column) and occluded (Right Column). 


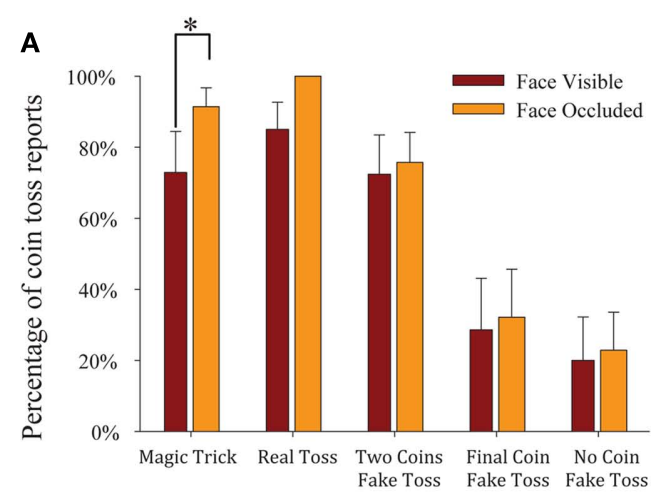

Free Viewing

B

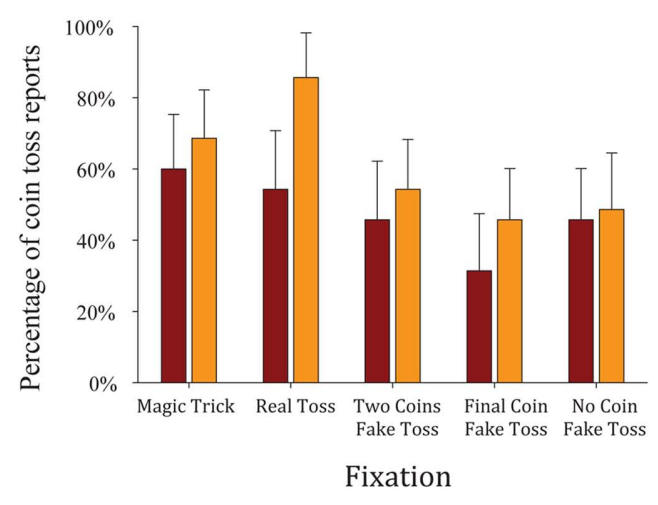

FIGURE 5 | Average percentage of coin toss reports according to type of performance. (A) Free-viewing condition. (B) Fixation condition. Error bars represent SEM across subjects $(N=8)$. ${ }^{*}$ Paired $t$-test, $p<0.05$.

Videos S1 and S2 in Supplementary Material). Thus, Experiment 3 introduced two new conditions in which the magician conducted simulated tosses from his empty right hand (Final Coin Fake Toss and No Coin Fake Toss). Figures 6B,C shows that the presence of an initial coin in the magician's hand resulted in higher percentages of coin toss reports, as predicted.

We wondered if the increased coin toss reports associated to the presence of an initial coin were potentially due to the flight of an actual coin in the Real Toss condition. To rule out this possibility, we repeated the analyses from Figures 6B,C after excluding the data from the Real Toss condition. We found that the presence of an initial coin resulted in higher percentages of coin toss reports, even in the absence of a genuine flying coin (data not shown).

\section{Effect of viewing condition}

We also wondered if the type of viewing condition (fixation versus free-viewing) might affect differentially the subjects' perception. The fixation condition required the subjects to look at a point in between the magician's eyes (or the equivalent position on the screen when the magician's face was blocked). Thus the subjects were forced to view the coin toss peripherally (and moreover were potentially susceptible to the magician's gaze misdirection when his face was visible). In contrast, the free-viewing condition
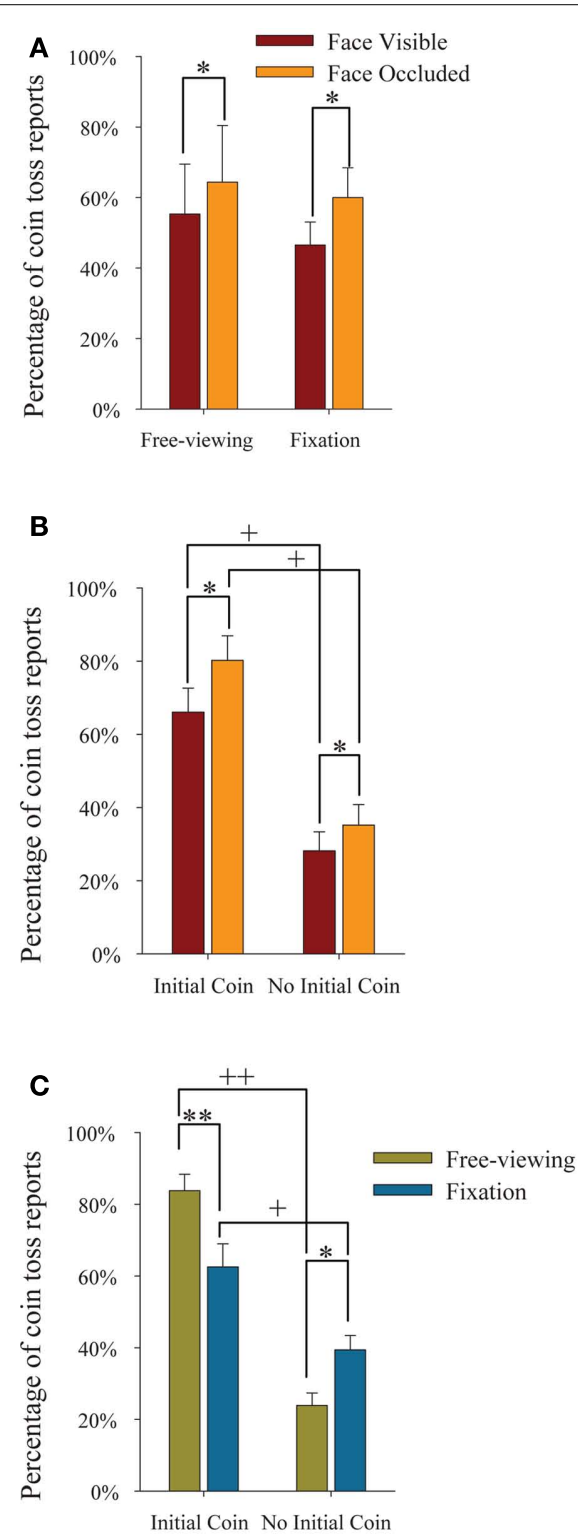

FIGURE 6 | Average percentage of coin toss reports according to viewing condition, presence of an initial coin, and presence/absence of social misdirection. (A) Percentages of button presses for free-viewing and fixation conditions, with the magician's face visible versus occluded. Error bars represent SEM across all five types of performance $(N=5$ conditions). (B) Percentages of button presses for types of performance in which an initial coin was present versus absent, with the magician's face visible versus occluded. Error bars under Initial Coin condition represent SEM across six conditions, i.e., Face visible and Face occluded, for Magic Trick, Real Toss and Two-Coin Fake Toss. Error bars under No Initial Coin condition represent SEM across four conditions, i.e., Face visible and Face occluded, for Final Coin Fake Toss and No Coin Fake Toss. (C) Percentages of button presses according to the presence/absence of an initial coin, with free-viewing versus fixation conditions. Error bars under Initial Coin condition represent SEM across six conditions, i.e., Free-viewing and Fixation, for Magic Trick, Real Toss and Two-Coin Fake Toss. Error bars under No Initial Coin condition represent SEM across four conditions, i.e., Free-viewing and Fixation, for Final Coin Fake Toss and No Coin Fake Toss. *Paired $t$-test, $p<0.05$; * Paired $t$-test, $p<0.001$; +Two-sample $t$-test, $p<0.05 ;++$ Two-sample $t$-test, $p<0.001$. 

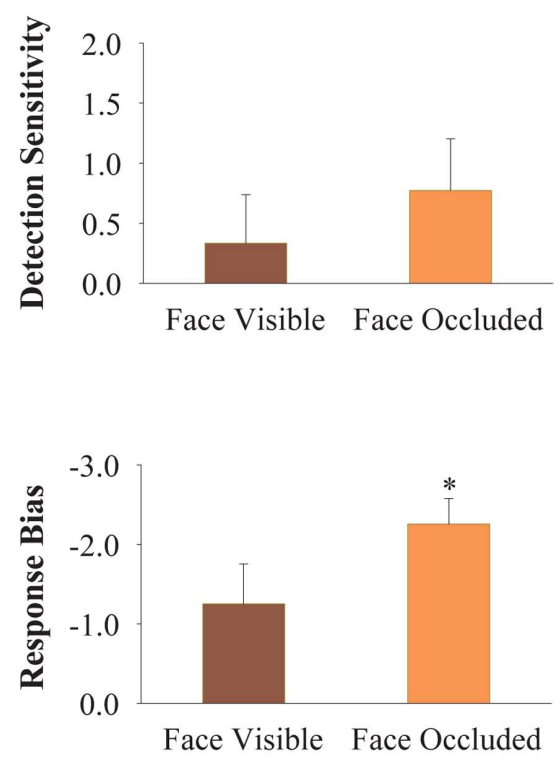

FIGURE 7 | Signal detection analyses of the effect of face visibility, presence of an initial coin and viewing task. Left Column: Detection sensitivity and response bias of Face Visible versus Face Occluded
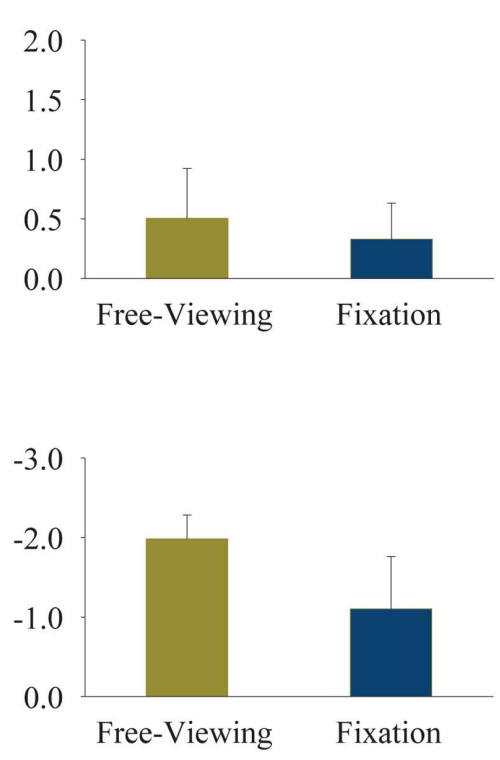

conditions. Right Column: Detection sensitivity and response bias of Free-viewing versus Fixation conditions. Error bars indicate SEM across subjects. *Paired $t$-test, $p<0.05$. allowed subjects to look anywhere on the screen. Figure 6C shows that free-viewing increased the percentages of coin toss reports only when an initial coin was present.

Detection sensitivity and response bias were comparable for the free-viewing and fixation conditions (Figure 7).

\section{Gaze dynamics}

We studied the subjects' gaze dynamics during the free-viewing of each video clip (Figure 8). In each type of performance, subjects tended to avoid the magician's face during the coin toss, focusing on the magician's hands instead. In most instances, subjects looked at the magician's face only after the time of the button press (usually waiting until the opening of the left hand).

Figure 9 further analyzes the subjects' gaze location in two time windows, one $500 \mathrm{~ms}$ before and another $500 \mathrm{~ms}$ after the button press. Unsurprisingly, subjects looked at the magician's face region more often when the face was visible than when it was occluded. No significant difference was observed between the gaze allocation before and after the button press, however. Moreover, subjects looked at the magician's face around the time of the button press only rarely (probability $<0.20$ ).

\section{DISCUSSION}

THE EFFECT OF SOCIAL MISDIRECTION IN THE PERCEPTION OF MAGIC

Joint attention is the mechanism by which an observer can share the experience of another by following his/her gaze direction and pointing gestures. Magicians rely on joint attention as a form of social misdirection, to direct the spectators' attention away from the method behind the magical effect, and toward the magical effect itself. If the magician wants the spectators' eyes (and/or attentional spotlight) focused on his face, he may look directly at his audience. If the magician instead wishes the spectators to shift their gaze (and/or attention) to a particular object, he himself may turn his head and eyes toward that object, and the heads and eyes (and/or attention) of the spectators will quickly follow suit (Macknik et al., 2008). Thus, the face of the magician provides effective social misdirection - via joint attention - in many magic illusions. Joint attention is critical for language acquisition and cognitive and social development (Scaife and Bruner, 1975; Tomasello and Farrar, 1986). But it also makes us susceptible to magic tricks that exploit our natural impulse to pay attention to the same places and objects attended by other people around us.

Previous research has found that magicians can effectively use their own direction of gaze to influence the gaze direction of observers (Kuhn and Land, 2006). Here we wondered if gaze misdirection could similarly improve the perception of a magic illusion not previously studied in the laboratory, which involves the simulated toss of a coin from hand to hand, and its subsequent perceptual vanish. In contrast to the previous studies (Kuhn and Land, 2006), we found that the magician's gaze misdirection did not intensify the subjects' perception of the illusion. Our data suggest that there is no simple "one size fit all" solution concerning the effects of social misdirection on the perception of magic, and that different magic illusions may be enhanced, unchanged, or lessened by social misdirection, in ways that remain to be explored.

The specific types of social misdirection studied may additionally explain some of the discrepancy between the current findings and Kuhn and Land's (2006). In Kuhn and Land's study, the magician's fake throw was conducted under two conditions of "social cueing": a pro-illusion condition in which the magician's eyes and head followed an imaginary ball moving upward, and an antiillusion condition, in which the magician looked at the hand concealing the ball. In the current study, the magician either looked 


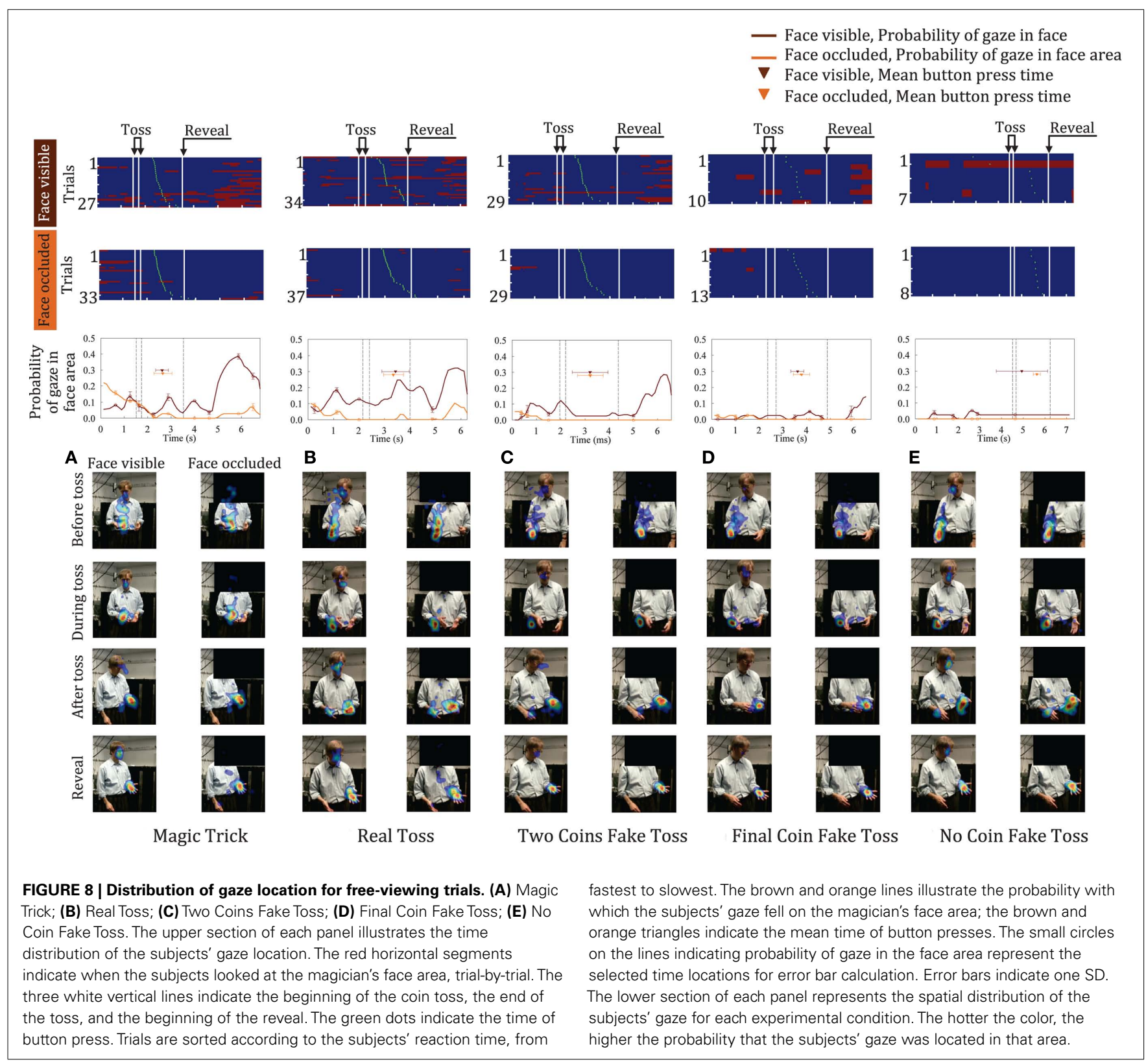

at the camera or his head was blocked. These two conditions are complementary to the ones used by Kuhn and Land, in that their magician looked either to the simulated position of the ball, or to the actual position of the ball, thus the magician's gaze was never neutral with respect to the ball. The inclusion of a "socially neutral" gaze condition could have involved the magician closing his eyes, or blocking the magician's eyes and head (as in the current study). Future research should ideally combine both experimental approaches, incorporating pro-illusion and anti-illusion conditions, but also conditions that eliminate or neutralize the potential effects of social misdirection.

The proficiency of the magician performing this trick may be a factor. Magic theorist Ascanio (2005) stated that a magician should strive for such degree of dexterity that no misdirection should be necessary, and such effective misdirection as to negate the need for high dexterity. Mac King may perform this sleight with such skillfulness that the illusion is already optimized without the addition of social misdirection (Max Maven, personal communication). Cavina-Pratesi et al. (2011) found that the kinematics of a magician's simulated grasp are very close to those of an actual grasp, and that magicians' simulated actions do not show many of the typical kinematic biases usually seen with pantomimed actions by non-magicians. Here we found that naive observers required a large amount of exposure to real and simulated coin tosses to distinguish most effectively between the two (20 trials combined; Figures 1 and 2). Our video recordings of Mac King performing real and simulated tosses moreover indicate that Mac King's timing of a simulated toss matches the timing of a real toss with great 


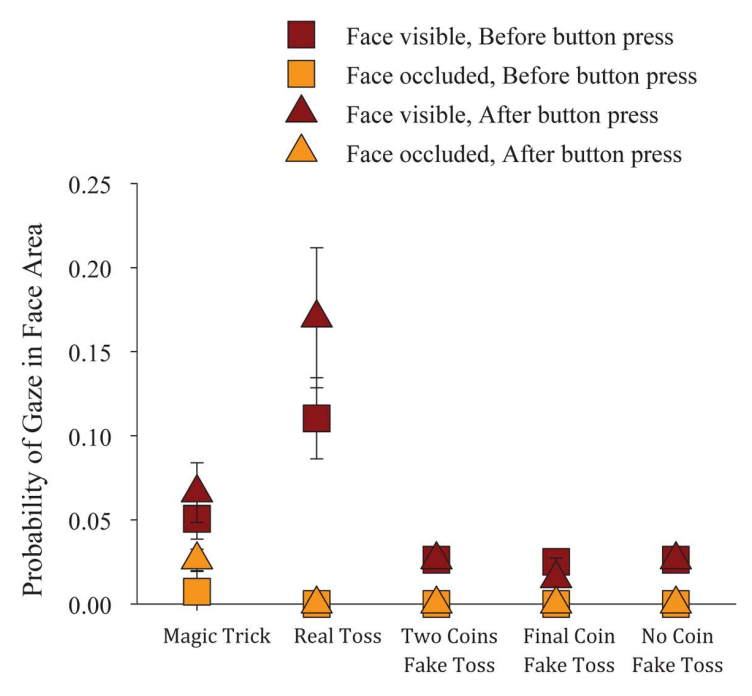

FIGURE 9 | Probability of the subjects' gaze on the magician's face area $\mathbf{5 0 0} \mathbf{~ m s}$ before versus $\mathbf{5 0 0} \mathbf{~ m s}$ after the button press. Error bars indicate one SD.

accuracy ( $\sim 235 \mathrm{~ms}$ in the Magic Trick condition, $\sim 269 \mathrm{~ms}$ in the Real Toss condition). Thus, one might conclude that social misdirection is redundant for this particular magic trick, but only if performed by a master magician.

One counterargument is that subjects were able to overcome the illusion eventually, despite Mac King's mastery. Around the 10th trial, Mac King's sleight-of-hand technique was no longer sufficient to maintain the illusion, whether his face was covered or not. Had the facial cues been effective as misdirection, the illusion would have persisted for more repetitions in the Face Visible condition than in the Face Occluded condition. This was not the case. It follows that if a less skilled magician were to perform this trick with imperfect sleight-of-hand, facial cues may not enhance the illusion either. If so, one practical recommendation to magicians performing this sleight would be to execute the toss without lifting their eyes to the audience, but to keep their gaze trained on the supposed location of the coin at any given time, so as to maximize the audience's attention to the illusory coin (via joint attention) and thus enhance the feeling of magic when the coin "vanishes" from the conjuror's hand. Future research should determine the effectiveness of social misdirection for this and other magic illusions, as executed by magicians with varying degrees of ability.

The current study focused on gaze misdirection as a potentially powerful source of joint attention. Future work should

\section{REFERENCES}

Ascanio, A. (2005). The Magic of Ascanio: The Structural Conception of Magic. New York: Paginas.

Bour, L. J., Aramideh, M., and De Visser, B. W. O. (2000). Neurophysiological aspects of eye and eyelid movements during blinking in humans. $J$. Neurophysiol. 83, 166-176.
Cavina-Pratesi, C., Kuhn, G., Ietswaart, M., and Milner, A. D. (2011). The magic grasp: motor expertise in deception. PLoS ONE 6, e16568. doi:10.1371/journal.pone. 0016568

Harvery, L. O. (2011). RscorePlus. Available at: http://psych.colorado.edu/ (lharvey/ [accessed; Online]. address the comparative effectiveness of the various forms of joint attention/social misdirection (i.e., gaze/head direction, body orientation, verbal cues, etc.) on the perception of this and other magic illusions.

\section{DO NOT DO THE SAME TRICK 10 TIMES}

The magician's axiom "Never do the same trick twice" indicates that if a magician were to perform the same trick twice for the same audience, there would be an increased chance that the audience would identify the underlying method and figure out the trick (King, 2007; Macknik et al., 2008). Several previous studies have shown that magic tricks are more likely to fail when observers view them a second time (Kuhn and Tatler, 2005; Kuhn and Land, 2006; Tatler and Kuhn, 2007; Kuhn et al., 2008). Similarly, many inattentional blindness demonstrations are a one-time only kind of effect. For instance, observers are more likely to detect a gorilla among basketball players if they watch the video for a second time (Simons and Chabris, 1999; Simons, 2010). Our current results indicate that some magic tricks are very resistant to repeated viewing, requiring many more than two performances to lose their effectiveness entirely.

\section{ACKNOWLEDGMENTS}

We thank Andrew Danielson for technical assistance, and Max Maven for very helpful discussions. This study was supported by the Barrow Neurological Foundation (Stephen L. Macknik and Susana Martinez-Conde) and the National Science Foundation (award 0852636 to Susana Martinez-Conde). Jorge Otero-Millan is a Fellow of the Pedro Barrié de la Maza Foundation.

\section{SUPPLEMENTARY MATERIAL}

The Supplementary Material for this article can be found online at http://www.frontiersin.org/Human_Neuroscience/10.3389/fnhum. 2011.00103/abstract

Video S1 | Magic trick.

Video S2 | Real toss.

Video S3 | Two-coin fake toss.

Video S4 | Final coin fake toss.

Video S5 | No coin fake toss.

Video S6 | Magic trick without reveal.

Video S7 | Real toss without reveal.

Hubel, D. H. (1988). Eye, Brain, and Vision. New York: Scientific American Library.

King, M. (2007). "It's a bad idea to do the same trick twice - and why magicians never announce their tricks in advance," in 11th Annual Meeting of the Association for the Scientific Study of Consciousness (Las Vegas).
Kuhn, G., and Land, M. F. (2006). There's more to magic than meets the eye. Curr. Biol. 16, R950-R951.

Kuhn, G., and Tatler, B. W. (2005). Magic and fixation: now you don't see it, now you do. Perception 34, 1155-1161.

Kuhn, G., Tatler, B. W., Findlay, J. M., and Cole, G. G. (2008). 
Misdirection in magic: implications for the relationship between eye gaze and attention. Vis. Cogn. 16, 391-405.

Macknik, S. L., King, M., Randi, J., Robbins, A., Teller, Thompson, J., and Martinez-Conde, S. (2008). Attention and awareness in stage magic: turning tricks into research. Nat. Rev. Neurosci. 9, 871-879.

Macknik, S. L., Martinez-Conde, S., and Blakeslee, S. (2010). Sleights of Mind. New York: Henry Holt and Company.

Macmillan, N. A., and Creelman, C. D. (2005). Detection Theory: A User's Guide. Mahwah, NJ: Lawrence Erlbaum Associates.

Marquardt, D. W. (1963). An algorithm for least-squares estimation of nonlinear parameters. J. Soc. Ind. Appl. Math. 11, 431-441.

Martinez-Conde, S., and Macknik, S. L. (2008). Magic and the brain. Sci. Am. 299, 72-79.

Press, W. H. (2002). Numerical Recipes in C++: The Art of Scientific Computing. Cambridge, NY: Cambridge University Press.

Scaife, M., and Bruner, J. S. (1975). Capacity for joint visual attention in infant. Nature 253, 265-266.

Simons, D. J. (2010). Monkeying around with the gorillas in our midst: familiarity with an inattentionalblindness task does not improve the detection of unexpected events. Perception 1, 3-6.
Simons, D. J., and Chabris, C. F. (1999). Gorillas in our midst: sustained inattentional blindness for dynamic events. Perception 28, 1059-1074.

Tatler, B. W., and Kuhn, G. (2007). "Don't look now: The magic of misdirection," in Eye Movements: A Window on Mind and Brain, eds R. P. G. Van Gompel, M. H. Fischer, W. S. Murray, and R. L. Hill (Oxford: Elsevier), 697-714.

Tomasello, M., and Farrar, M. J. (1986). Joint attention and early language. Child Dev. 57, 1454-1463.

Conflict of Interest Statement: The authors declare that the research was conducted in the absence of any commercial or financial relationships that could be construed as a potential conflict of interest.

Received: 10 April 2011; accepted: 05 September 2011; published online: 29 September 2011.

Citation: Cui J, Otero-Millan J, Macknik SL, King $M$ and MartinezConde S (2011) Social misdirection fails to enhance a magic illusion. Front. Hum. Neurosci. 5:103. doi: 10.3389/fnhum.2011.00103

Copyright (C) 2011 Cui, Otero-Millan, Macknik, King and Martinez-Conde. This is an open-access article subject to a non-exclusive license between the authors and Frontiers Media SA, which permits use, distribution and reproduction in other forums, provided the original authors and source are credited and other Frontiers conditions are complied with. 